\title{
HIGHER-DIMENSIONAL HEREDITARILY INDECOMPOSABLE CONTINUA
}

\author{
BY
}

R. H. BING

1. Introduction and definitions. A continuum is indecomposable if it is not the sum of two proper subcontinua. It is hereditarily indecomposable if each of its subcontinua is indecomposable. Knaster gave [5](1) an example of a 1-dimensional hereditarily indecomposable continuum. Moise described [6] a plane hereditarily indecomposable continuum (it was topologically equivalent [2] to the one described by Knaster) which was topologically equivalent to each of its nondegenerate subcontinua. I showed [1] that the same con-

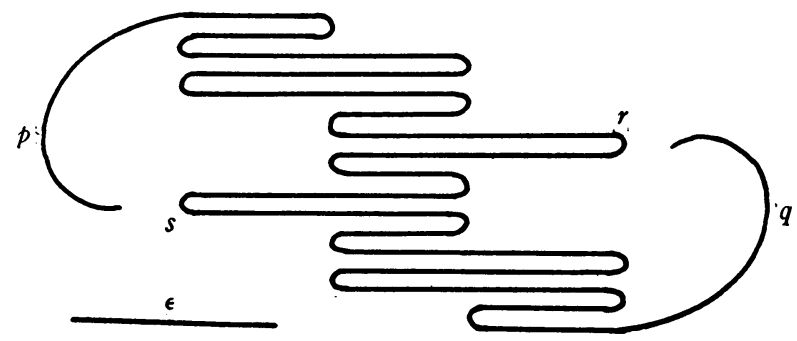

FIG. 1

tinuum was homogeneous. Recently, I gave [2] examples of many topologically different hereditarily indecomposable continua. However, each of these continua was 1 -dimensional. A point is a 0 -dimensional hereditarily indecomposable continuum. (In this paper we define a continuum to be a compact closed connected set.)

Kelly has shown [4] that if there is a hereditarily indecomposable continuum of dimension more than one, there is one of infinite dimension. In this paper we show that there are hereditarily indecomposable continua of all dimensions. In describing these continua, we make use of the following term.

$\epsilon$-crooked. An arc is $\epsilon$-crooked if for each pair of its points $p$ and $q$ there are points $r$ and $s$ between $p$ and $q$ on the arc such that $r$ lies between $p$ and $s$, $\rho(p, s)<\epsilon$, and $\rho(r, q)<\epsilon$ where $\rho(x, y)$ denotes the distance between $x$ and $y$. See Fig. 1. It may be noted that if $\epsilon^{\prime}>\epsilon$, an arc is $\epsilon^{\prime}$-crooked if it is $\epsilon$-crooked. An arc intersecting sets $H$ and $K$ is called $\epsilon$-crooked with respect to them if each of its subarcs $p q$ with ends $p$ and $q$ on $H$ and $K$ respectively contains

Presented to the International Congress of Mathematicians, September 6, 1950; received by the editors February 21, 1951.

(1) Numbers in brackets refer to the references cited at the end of the paper. 
points $r$ and $s$ such that $r$ lies between $p$ and $s$ on $p q, \rho(s, H)<\epsilon$, and $\rho(r, K)$ $<\epsilon$.

We use $E_{n}$ to denote an $n$-dimensional Euclidean space and $I_{\omega}$ to denote a Hilbert cube. The points of a Hilbert cube are countable sequences $\left(x_{1}, x_{2}, \cdots\right)$ such that $\left|x_{i}\right| \leqq 1 / i$ and the distance between two points is given by $\rho(x, y)$ $=\sum\left|x_{i}-y_{i}\right| / 2^{i}$. We make use of the facts that $I_{\omega}$ is compact and any separable metrizable space can be topologically imbedded in $I_{\omega}[3, \mathrm{p} .64]$. Throughout this paper we restrict ourselves to metric spaces.

2. Description of a 2-dimensional hereditarily indecomposable continuum. We shall show that if $b$ and $c$ are two points of $E_{3}$, there is a sequence $S_{1}, S_{2}, \ldots$ of bounded connected domains satisfying the following conditions:

(1) $S_{i}$ contains $\bar{S}_{i+1}$.

(2) $S_{i}$ separates $b$ from $c$.

(3) $E_{3}-S_{i}$ has only two components and no point of $S_{i}$ is farther than $1 / i$ from either of them.

(4) Each arc in $S_{i}$ is $1 / i$-crooked.

The intersection $S_{0}$ of $S_{1}, S_{2}, \cdots$ is a 2 -dimensional hereditarily indecomposable continuum which has exactly two complementary domains and which is irreducible with respect to separating $E_{3}$.

We may take $S_{1}$ to be the set of all points $p$ of $E_{3}$ such that minimum $(1 / 4, \rho(b, c) / 3)<\rho(b, p)<$ minimum $(1 / 2,2 \rho(b, c) / 3)$. That $S_{2}, S_{3}, \cdots$ may be chosen so as to satisfy conditions (1), (2), (3), and (4) follows from Theorem 2.

Since, by condition (1), $S_{0}=\bar{S}_{1} \cdot \bar{S}_{2} \cdots$, it is a continuum. We find from condition (2) that $S_{0}$ separates $b$ from $c$ and from condition (3) that $S_{0}$ has only two complementary domains and is irreducible with respect to separating $E_{3}$. That $S_{0}$ is 2-dimensional follows from the fact that it is irreducible with respect to separating $E_{3}$.

Condition (4) insures that $S_{0}$ is hereditarily indecomposable. To see that this is true, assume that $H$ and $K$ are two intersecting continua in $S_{0}$ such that neither $H$ nor $K$ contains the other. There would be a positive integer $n$ and points $p$ and $q$ of $H$ and $K$ respectively such that $\rho(p, K)>1 / n$ and $\rho(q, H)>1 / n$. Let $O_{H}$ and $O_{K}$ be connected open subsets of $S_{n}$ containing $H$ and $K$ respectively such that $\rho\left(p, O_{K}\right)>1 / n$ and $\rho\left(q, O_{H}\right)>1 / n$. Let $p x q$ be an arc from $p$ to $q$ in $O_{H}+O_{K}$ such that $O_{H}$ and $O_{K}$ contain $p x$ and $x q$ respectively. Since $\rho(p, x q)>1 / n$ and $\rho(p x, q)>1 / n, p x q$ is not $1 / n$-crooked. The assumption that $S_{0}$ is not hereditarily indecomposable has led to the contradiction that an arc in $S_{n}$ is not $1 / n$-crooked.

3. Crooked domains. The following two theorems hold in either $E_{n}$ or in $I_{\omega}$.

Theorem 1. Suppose $D$ is a bounded domain that separates the point $b$ from the point $c, H$ and $K$ are two point sets, and $\epsilon$ is a positive number. Then there 
is a domain $E$ in $D$ that separates $b$ from $c$ such that each arc in $E$ from a point of $H$ to a point of $K$ is $\epsilon$-crooked with respect to $H$ and $K$.

Proof. Let $K^{\prime}=\{x \mid x$ element of $K, \rho(x, H) \geqq \epsilon\}$ where the symbol $\{x \mid \mathrm{P}\}$ is used to denote the collection of all points $x$ such that $x$ satisfies condition $\mathrm{P}$. We suppose that neither $K^{\prime}$ nor $H$ is empty because if either is, we can let $E$ be $D$.

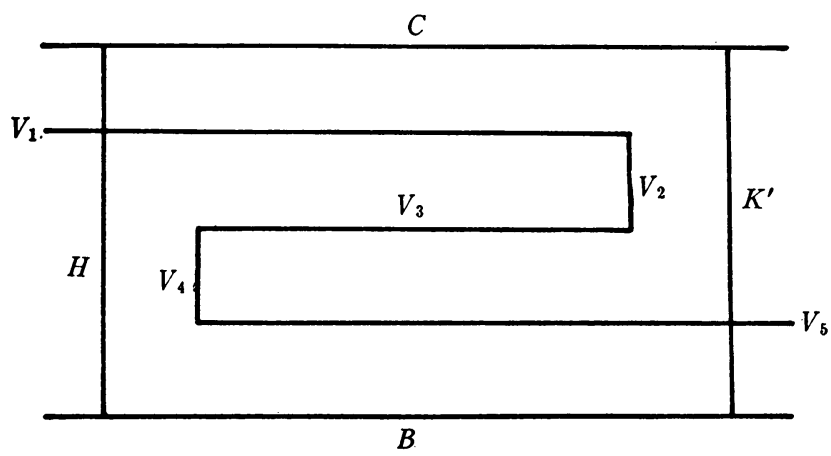

FIG. 2

Suppose the complement of $D$ is the sum of the mutually exclusive closed sets $B$ and $C$ which contain $b$ and $c$ respectively, and that $\delta$ is a positive number less than the distance from any point of $B$ to any point of $C$. We define $V$ to be the sum of the following five sets $V_{1}, V_{2}, \cdots, V_{5}$.

$$
\begin{aligned}
& V_{1}=\left\{x \mid \rho(x, B)=3 \delta / 4, \rho\left(x, K^{\prime}\right) \geqq \epsilon / 2\right\} . \\
& V_{2}=\left\{x \mid \delta / 2 \leqq \rho(x, B) \leqq 3 \delta / 4, \rho\left(x, K^{\prime}\right)=\epsilon / 2\right\} . \\
& V_{3}=\left\{x \mid \rho(x, B)=\delta / 2, \rho\left(x, H+K^{\prime}\right) \geqq \epsilon / 2\right\} . \\
& V_{4}=\{x \mid \delta / 4 \leqq \rho(x, B) \leqq \delta / 2, \rho(x, H)=\epsilon / 2\} . \\
& V_{5}=\{x \mid \rho(x, B)=\delta / 4, \rho(x, H) \geqq \epsilon / 2\} .
\end{aligned}
$$

We find that $V$ separates $B$ from $C$ (see Fig. 2) because it contains the boundary of the closed set

$$
\begin{aligned}
\{x \mid \rho(x, B) \leqq \delta / 4\} & +\{x \mid \delta / 4 \leqq \rho(x, B) \leqq \delta / 2, \rho(x, B) \leqq \epsilon / 2\} \\
& +\left\{x \mid \delta / 2 \leqq \rho(x, B) \leqq 3 \delta / 4, \rho\left(x, K^{\prime}\right) \geqq \epsilon / 2\right\}
\end{aligned}
$$

which contains $B$ but no point of $C$.

Let $E, E_{1}, E_{2}, \cdots, E_{5}$ be $\gamma$ neighborhoods of $V, V_{1}, V_{2}, \cdots, V_{5}$ respectively where $\gamma$ is the minimum of $\epsilon / 4$ and $\delta / 16$. We note that $\bar{E}$ lies in $D$. Also, $E$ separates $b$ from $c$ because it contains $V$. No point of $E_{4}$ is as far as $\epsilon$ from $H$ and no point of $E_{2}$ is as far as $\epsilon$ from $K$.

We shall show that if $p q$ is an arc in $E$ from a point $p$ of $H$ to a point $q$ of 
$K$, there are points $r$ and $s$ on $p q$ between $p$ and $q$ such that $r$ is between $p$ and $s, \rho(s, H)<\epsilon$, and $\rho(r, K)<\epsilon$. If $q$ is nearer than $\epsilon$ to $H, r$ and $s$ may be found near $q$. If $q$ is a point of $K^{\prime}, q$ belongs to $E_{5}$ and $p$ belongs to $E_{1}$. In Fig. 2, $E$ would be a small neighborhood of $V$. Since $E_{2}$ separates $E \cdot H$ from $E_{4}$ and $E_{4}$ separates $E_{2}$ from $E \cdot K^{\prime}$, there are points $r$ and $s$ of $p q$ belonging to $E_{2}$ and $E_{4}$ respectively such that $r$ is between $p$ and $s$ on $p q$. Hence, any arc in $E$ from a point of $H$ to a point of $K$ is $\epsilon$-crooked with respect to $H$ and $K$.

THEOREM 2. If $D$ is a bounded domain that separates the point $b$ from the point $c$ and $\epsilon$ is a positive number, there is a connected domain $E$ satisfying the following conditions:

(1) D contains $\bar{E}$.

(2) E separates $b$ from $c$.

(3) The complement of $E$ has exactly 2 components and no point of $E$ is farther than $\epsilon$ from either of them.

(4) Each arc in $E$ is $\epsilon$-crooked.

Proof. Suppose $G$ is a finite covering of $D=D_{0}$ by sets of diameter less than $\epsilon / 2$. Let $\left(g_{1}, g_{1}^{\prime}\right),\left(g_{2}, g_{2}^{\prime}\right), \cdots,\left(g_{n}, g_{n}^{\prime}\right)$ be the finite collection of pairs of elements of $G$. By repeated applications of Theorem 1 we find that there is a domain $D_{i}(i=1,2, \cdots, n)$ such that $\bar{D}_{i}$ lies in $D_{i-1}, D_{i}$ separates $b$ from $c$, each arc in $D_{i}$ from $g_{i}$ to $g_{i}^{\prime}$ is $\epsilon / 2$-crooked with respect to $g_{i}$ and $g_{i}^{\prime}$. The domain $D_{n}$ satisfies conditions (1), (2), and (4). Since $E_{n}$ and $I_{\omega}$ are unicoherent, one component of $D_{n}$ separates $b$ from $c$. By subtracting the sum of a collection of arcs from this component we may obtain a connected domain $E$ satisfying conditions (1), (2), (3), and (4).

4. Higher-dimensional hereditarily indecomposable continua. Using the same scheme used in $\$ 2$ to define $S_{0}$ in $E_{3}$, we can define hereditarily indecomposable continua in $E_{n}$ and in $I_{\omega}$. The following result holds in either $E_{n}$ or $I_{\omega}$.

THEOREM 3. If $H$ and $K$ are two mutually exclusive continua, there is a hereditarily indecomposable continuum which has exactly two complementary domains and which is irreducible with respect to separating $H$ from $K$.

Using Theorem 3, we can get the following result which shows that there are hereditarily indecomposable continua of all dimensions.

THEOREM 4. There are infinite-dimensional hereditarily indecomposable continua in $I_{\omega}$ and there are $n$-dimensional hereditarily indecomposable continua in $E_{n+1}$.

THEOREM 5. Each $n+1$-dimensional continuum contains an $n$-dimensional hereditarily indecomposable continuum.

Proof. If $M$ is an $n+1$-dimensional continuum, it contains two mutually exclusive closed subsets $B$ and $C$ such that no closed subset of $M$ of dimen- 
sion less than $n$ separates $B$ from $C[3$, p. 26]. We find from Theorem 6 of the next section that there is a closed subset $H$ of $M$ which separates $B$ from $C$ such that each component of $H$ is hereditarily indecomposable. One component $C$ of $H$ is not of dimension less than $n$ [3, p. 94]. There is an $n$-dimensional subcontinuum of $C$ and this subcontinuum is hereditarily indecomposable because $C$ is.

5. Separating with collections of hereditarily indecomposable continua. In this section we consider separations by closed point sets such that the components of these closed point sets are hereditarily indecomposable.

Theorem 6. If $B$ and $C$ are two mutually exclusive closed subsets of a continuum $M$, there is a closed point set $H$ in $M$ separating $B$ from $C$ in $M$ such that each component of $H$ is hereditarily indecomposable.

Froof. There is a continuous transformation $T$ of $M$ into $I_{\omega}$ such that $T(B)$ is a point, $T(C)$ is another point, and $T$ is $1-1$ on $M-(B+C)$. We find from Theorem 3 that there is a hereditarily indecomposable continuum $K$ in $I_{\omega}$ which separates $T(B)$ from $T(C)$. Then $H=T^{-1}[K \cdot T(M)]$.

The following result can be obtained in a similar fashion.

Theorem 7. If $B$ and $C$ are two mutually exclusive bounded closed subsets of $E_{n}$, there is a closed bounded point set $H$ separating $B$ from $C$ such that each component of $H$ is hereditarily indecomposable.

THEOREM 8. If $p$ and $q$ are two points of $E_{n}$, there is a bounded closed point set $H$ in $E_{n}$ separating $p$ from $q$ such that each continuum in $E_{n}$ containing $p+q$ contains a component of $H$. it.

Before proving Theorem 8 we state the following interesting corollary of

Corollary. For each pair of points $p, q$ of $E_{n}$ there is a bounded closed set $H$ in $E_{n}-(p+q)$ such that each arc from $p$ to $q$ in $E_{n}$ contains a degenerate component of $H$.

Proof of Theorem 8. Suppose $E_{n}$ is a plane in $E_{n+1}$. Then $H$ is the intersection of $E_{n}$ with a hereditarily indecomposable continuum $K$ in $E_{n+1}$ which separates $p$ from $q$ in $E_{n+1}$.

We shall show that the assumption that there is a continuum $C$ in $E_{n}$ which contains $p+q$ but no component of $H$ leads to the contradiction that $K$ contains a decomposable continuum.

Suppose there is such a continuum $C$ in $E_{n}$. We now show that there is a unicoherent locally connected continuum $M$ in $E_{n+1}$ such that $M \cdot E_{n}=C$. Let $P$ be a compact locally connected continuum in $E_{n}$ containing $C$ and $r$ be a point whose distance from $E_{n}$ is greater than the diameter of $P$. We define $M=\{x \mid x$ lies on closed interval between $r$ and a point $p(x)$ of $P, \rho[x, p(x)]$ 
$\geqq \rho[p(x), C]\}$.

If $M^{\prime}$ is the cone with vertex $r$ and base $P$, we may think of $M$ as the image of $M^{\prime}$ under a homeomorphism that moves toward $r$ those points of the cone $M^{\prime}$ which are not between $r$ and $C$. Since $M^{\prime}$ is a cone whose base is a locally connected continuum, $M^{\prime}$ is locally connected and unicoherent. Hence, $M$ is also.

Since $M$ is a locally connected continuum and $M \cdot K$ separates $p$ from $q$ in $M$, some closed subset $S_{1}$ of $M \cdot K$ is irreducible with respect to separating $p$ from $q$. Since $M$ is unicoherent, $S_{1}$ is connected. In order for $S_{1}$ to separate $p$ from $q$ in $M$ it must intersect $C$ but must not be a subset of $E_{n}$. Let $s$ be a point of $S_{1} \cdot E_{n}$ and $S_{2}$ be the component of $H$ that contains $s$. Now $S_{2}$ does not lie in $S_{1}$ because $C$ contains no component of $H$ and $S_{1}$ does not lie in $S_{2}$ because it is not a subset of $E_{n}$. Hence the assumption that there is a continuum $C$ in $E_{n}$ which contains $p+q$ but no component of $H$ has led to the contradiction that the hereditarily indecomposable continuum $K$ contains the decomposable continuum $S_{1}+S_{2}$.

THEOREM 9. For each pair of points $p, q$ of a continuum $M$ there is a closed set $H$ in $M-(p+q)$ such that each subcontinuum of $M$ containing $p+q$ contains a component of $H$.

Proof. The proof is similar to that used in Theorem 8. We suppose $M$ lies in a hyperplane in $I_{\omega}$ and let $H$ be the intersection of $M$ with a hereditarily indecomposable continuum in $I_{\omega}$ that separates $p$ from $q$.

6. Homogeneity. A set $M$ is homogeneous if for each pair of its points $p, q$ there is a homeomorphism of $M$ into itself that carries $p$ into $q$. I used [1] a pseudo-arc [6] (a nondegenerate hereditarily indecomposable continuum that can be chained [2]) to show that the simple closed curve is not the only type of nondegenerate homogeneous plane continuum. However, we find from the following result that if $n$ is an integer greater than one, no $n$-dimensional hereditarily indecomposable continuum is homogeneous.

THEOREM 10. If $M$ is an n-dimensional hereditarily indecomposable continuum, there is a point $p$ such that each nondegenerate subcontinuum of $M$ containing $p$ is $n$-dimensional.

Proof. Suppose $M$ is the sum of a finite number of closed sets each of diameter less than 1 . One of the closed sets had dimension $n$ and one of its components has dimension $n$. Hence, $M$ has an $n$-dimensional subcontinuum $M_{1}$ of diameter less than 1 . Similarly, $M_{1}$ has an $n$-dimensional subcontinuum $M_{2}$ of diameter less than $1 / 2, M_{2}$ has an $n$-dimensional subcontinuum $M_{3}$ of diameter less than $1 / 3, \cdots$. If $p=M_{1} \cdot M_{2} \ldots$, each nondegenerate subcontinuum $C$ of $M$ containing $p$ is $n$-dimensional because it contains one of the $M_{i}$ 's. If $M_{i}$ does not contain $C, C$ contains $M_{i}$ because $C+M_{i}$ is hereditarily indecomposable. 


\section{ReferenCES}

1. R. H. Bing, A homomogeneous indecomposable plane continuum, Duke Math. J. vol. 15 (1948) pp. 729-742.

2. - Concerning hereditarily indecomposable continua, Pacific Coast Mathematical Journal vol. 1 (1951) pp. 43-51.

3. W. Hurewicz and H. Wallman, Dimension theory, Princeton University Press, 1948.

4. J. L. Kelly, The hyperspaces of a continuum, Trans. Amer. Math. Soc. vol. 52 (1942) pp. 22-36.

5. B. Knaster, Un continu dont tout sous-continu est indécomposable, Fund. Math. vol. 3 (1922) pp. 247-286.

6. E. E. Moise, An indecomposable plane continuum which is homeomorphic to each of its nondegenerate subcontinua, Trans. Amer. Math. Soc. vol. 63 (1948) pp. 581-594.

UNIVERSITY OF WisCONSIN,

Madison, Wis. 